Tropical Journal of Pharmaceutical Research October 2021; 20 (10): 2213-2218

ISSN: $1596-5996$ (print); 1596-9827 (electronic) (C) Pharmacotherapy Group, Faculty of Pharmacy, University of Benin, Benin City, 300001 Nigeria.

\title{
Therapeutic efficacy of a combination of mesalazine and Bifid Triple Viable Capsules (BTVCs) on ulcerative colitis patients, and its effect on inflammation and oxidative stress
}

\author{
Lina Wei, Hua Xu* \\ Department of Gastroenterology, The Second Affiliated Hospital of Dalian Medical University, Dalian 116023, Liaoning
} Province, China

*For correspondence: Email: x1k3fl@163.com; Tel: +86-0411-84671291

Sent for review: 10 July 2021

Revised accepted: 29 September 2021

\begin{abstract}
Purpose: To determine the curative impact of mesalazine (MSZ)-BTVCs combination on ulcerative colitis (UC), and its influence on inflammation and oxidative stress in the patients.

Methods: 100 UC patients were randomely assigned to a control group given MSZ capsule treatment only, and a combination group treated with BTVCs and MSZ. Treatment effectiveness, inflammatory response, and oxidative stress in the two groups before and after treatment were compared.

Results: The combination group had higher total effectiveness than the control group. The serum levels of MDA, high-sensitivity C-reactive protein (hs-CRP), TNF- $\alpha$ and interleukin-6 (IL-6) were lower, while serum levels of superoxide dismutase (SOD) and interleukin-10 (IL-10) were markedly increased in patients given combination treatment, when compared with controls. Pre-drug exposure UC disease activity index (UC-DAI) and clinical symptom scores were similar in both cohorts of patients, but the post-treatment scores were statistically decreased, especially in the combination group.

Conclusion: The combined use of MSZ and BTVCs was more effective against UC than monotherapy, as it effectively relieved inflammation and oxidative stress in patients, resulting in better clinical efficacy.
\end{abstract}

Keywords: MSZ, BTVCs, Ulcerative colitis, Inflammation, Oxidative stress

\begin{abstract}
This is an Open Access article that uses a funding model which does not charge readers or their institutions for access and distributed under the terms of the Creative Commons Attribution License (http://creativecommons.org/licenses/by/4.0) and the Budapest Open Access Initiative (http://www.budapestopenaccessinitiative.org/read), which permit unrestricted use, distribution, and
\end{abstract} reproduction in any medium, provided the original work is properly credited.

Tropical Journal of Pharmaceutical Research is indexed by Science Citation Index (SciSearch), Scopus, International Pharmaceutical Abstract, Chemical Abstracts, Embase, Index Copernicus, EBSCO, African Index Medicus, JournalSeek, Journal Citation Reports/Science Edition, Directory of Open Access Journals (DOAJ), African Journal Online, Bioline International, Open-J-Gate and Pharmacy Abstracts

\section{INTRODUCTION}

Ulcerative colitis (UC), a digestive disease with high incidence, is pervasive in young people aged $20-40$ years [1]. The improvement of living standards of people in recent years has driven the ongoing incidence of UC in China. The occurrence of UC is attributed to multiple factors, but its mechanism remains elusive at present. In light of its close relationship with inflammatory reactions, UC is considered an inflammatory disease [2]. Impaired scavenging of oxygen free radicals (OFR) in the body is also a key factor promoting ulcer formation, since increased levels of OFR enhance the activation of inflammatory mediators and induce an inflammatory response [3]. 
A positive microflora habitat in the intestinal microenvironment is conducive for the growth of large mass of microflora that maintains the mucosal barrier, aids digestion, and also influences various physiological processes in the body [4]. In recent years, a growing number of studies have linked this microenvironment to gastrointestinal diseases, since the gut microbiota is considered a vital organ of the body [5]. Increased levels of OFRs in patients lead to the activation of inflammatory mediators which in turn, enhance inflammatory reactions, damage tissues, and seriously affect the physical and mental health of patients. Currently, salicylic acid preparation [6] and sulfasalazine [7] are the main drugs used for the mitigation of inflammatory response in clinical practice. However, monotherapy of either drug usually fails to produce a satisfactory efficacy. Microecological preparation can bolster the intestinal environment, thereby increasing mucosal permeability which is of paramount importance in the treatment of UC.

Accordingly, this study applied combined use of MSZ and BTVCs for treating UC patients, and evaluated its curative effect and influence on inflammation and oxidative stress reaction in the patients.

\section{METHODS}

\section{Patient profile}

One hundred patients with UC who received inpatient or out-patient treatment in the Digestive System Department of our hospital from June 2019 to June 2020 were selected and allocated to combination group ( $\mathrm{n}=89$; male-to-female ratio: 49:40, mean age: $43.5 \pm 14.4$ years) and control group ( $n=11$; male-to-female ratio: 9:2; mean age: $42.4 \pm 19.2$ years) using the random number table method. All the enrolled patients were diagnosed with UC which was confirmed via colonoscopy, pathology and clinical manifestations, with the lesion located in the rectum or sigmoid colon. The participation by all patients was without compulsion, and all participants submitted signed statements indicating their consent. Patients with drug allergy, blood coagulation disorder, peptic ulcer, surgical history, medication history involving hormones or immune-boosting preparations, as well as pregnant and lactating women, were excluded. Baseline data was similar in both groups $(p>0.05)$. The research protocol was approved by the Hospital Ethics Committee (approval no. 1846819L). The protocol used in this study followed the World Medical Association Declaration of Helsinki [8].

\section{Treatments}

The control group was given mesalazine $(1 \mathrm{~g}$ at a time for a total of 4 times per day), while the combination group was, in addition, given oral administration of Bifid Triple Viable Capsules (420 mg half an hour after meals, twice for patients with a mild and moderate condition, and three times for patients with severe conditions. The course of treatment in both groups spanned 8 weeks.

\section{Assessment of clinical efficacy}

The clinical efficacy in patients was classified as highly effective, effective or ineffective. To be specific, highly effective indicated that after treatment, the clinical symptoms subsided; the intestinal mucosal ulcer tissue was healed, and stool test showed negative UC results. Effective indicated that after treatment, the clinical symptoms were significantly reduced; the intestinal mucosal ulcer tissue showed only light inflammation, while false polyp tissue could be seen, and the stool test result was negative. In contrast, ineffective indicated that after treatment, the clinical symptoms and intestinal mucosal ulcer tissue were not mitigated, and the stool test result was positive. Total response to treatment (TRT) was calculated as shown in Equation 1.

$\operatorname{TRT}(\%)=[(\mathrm{NS}+\mathrm{Ne}) / \mathrm{Nt}] \times 100$

where $N s=$ number of significantly effective cases, $\mathrm{Ne}=$ number of effective cases, and $\mathrm{Nt}=$ total number of cases.

\section{Determination of oxidative stress and inflammatory factors}

Before and after treatment, $5 \mathrm{ml}$ of elbow venous blood was collected from patients in both groups under fasting condition. The blood samples were centrifuged at $2000 \mathrm{rpm}$ for $10 \mathrm{~min}$ to obtain sera. Serum hs-CRP was determined using radio immunoturbidimetry, while serum levels of IL-6, TNF- $\alpha$ and IL-10 were assayed using ELISA. Serum superoxide dismutase (SOD) was determined using the nitrite method, while serum malondialdehyde (MDA) was determined using the thiobarbituric acid (TBA) method.

\section{Intestinal mucosal barrier function indicators}

D-Lactic acid (D-LA) level was determined using D-lactic acid Colorimetric Assay Kit provided by eBioscience Inc. (San Diego, USA; item no. EBC-K002-M). Diamine oxidase (DAO) was determined with Human Diamine Oxidase (DO) 
ELISA Kit provided by eBioscience, Inc. (San Diego, USA; item no. PKSH032342. Endotoxin (ET) was determined with Human Endothelin 1 (ET-1) ELISA kit provided by eBioscience, Inc (San Diego, USA; Item no. E-EL-H0064c).

\section{Evaluation of activity and clinical symptom score}

The UC disease activity index (UC-DAI) was applied for the evaluation of disease activity before and after treatment, and clinical symptom scores were utilized for assessment of changes in clinical symptoms such as abdominal pain, diarrhea and mucus bloody stool before and after treatment.

\section{Statistical analysis}

Statistical analysis was done via SPSS 21.0. Measurement data are presented as mean \pm SD. Comparison was done with independent $t$-test, while count data were analyzed using $x^{2}$ test. Significance of difference was assumed at $p<$ 0.05 .

\section{RESULTS}

\section{Overall efficacy}

As shown in Table 1, the combination group had a significantly higher overall efficacy (93.3\%) than the control group which had a value of 72.7 $\%(p<0.05)$.

\section{Oxidative stress indicators}

In both groups, SOD activity and MDA content were similar before treatment, while SOD activity increased and MDA content decreased after treatment. Compared with the control group, the reduction in MDA content and the elevation in SOD activity were more evident in the combination group $(p<0.05$; Figure 1$)$.

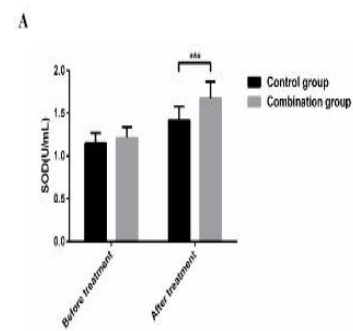

B

Figure 1: Comparison of oxidative stress indexes between two groups before and after treatment. (A) Comparison of SOD activity between the two groups before and after treatment; B: comparison of MDA content before and post-drug exposure. ${ }^{* *} P<0.001$, when compared to control

\section{Inflammatory factors}

Before treatment, levels of inflammatory factors (hs-CRP, TNF- $\alpha$, IL-6 and IL-10) were similar in the two groups. However, levels of levels of hsCRP, TNF- $\alpha$ and IL- 6 were decreased, while IL10 level was increased after treatment, with statistically significant differences between both groups (Table 2).

Table 1: Comparison of overall efficacy between the two groups

\begin{tabular}{lcccc}
\hline Group & Highly effective & Effective & Ineffective & Total effectiveness \\
\hline Control $(\mathrm{n}=11)$ & $5(45.4)$ & $3(27.3)$ & $3(27.3)$ & $8(72.7)$ \\
Combination $(\mathrm{n}=89)$ & $71(79.8)$ & $12(13.5)$ & $6(6.7)$ & $83(93.3)$ \\
$X^{2} / \mathrm{t}$ & & & & 5.0391 \\
$P$-value & & & 0.0248 \\
\hline
\end{tabular}

Table 2: Levels of inflammatory factors in both groups (pg/mL, except for hs-CRP which is in $\mathrm{mg} / \mathrm{mL}$ )

\begin{tabular}{lcccc}
\hline Variable & Control group $(\mathbf{n = 1 1})$ & Combination group $(\mathbf{n = 8 9})$ & $\boldsymbol{t}$ & $\boldsymbol{P}$-value \\
\hline hs-CRP & $78.62 \pm 9.26$ & $77.25 \pm 8.26$ & 0.5123 & 0.610 \\
Before & $41.25 \pm 5.26$ & $25.61 \pm 2.64$ & 16.2385 & $<0.001$ \\
After & & & & \\
TNF- $\alpha$ & $35.62 \pm 4.25$ & $34.25 \pm 3.91$ & 1.0863 & 0.280 \\
Before & $24.25 \pm 2.86$ & $16.82 \pm 1.94$ & 11.3246 & $<0.001$ \\
After & & & & \\
IL-6 & $197.62 \pm 22.51$ & $193.51 \pm 20.61$ & 0.6179 & 0.538 \\
Before & $91.62 \pm 9.05$ & $75.82 \pm 7.64$ & 6.3416 & $<0.001$ \\
After & & & & \\
IL-10 & $25.12 \pm 2.36$ & $26.01 \pm 2.45$ & 2.1663 & 0.257 \\
Before & $53.52 \pm 4.25$ & $73.82 \pm 7.36$ & 8.9383 & $<0.001$ \\
After & & & & \\
\hline
\end{tabular}


Indicators of intestinal mucosal barrier function

Table 3 shows that prior to treatment, no noteworthy difference was observed in intestinal mucosal barrier function between both groups, while post-treatment level of D-LA, DAO and ET were markedly reduced in combination group, relative to control.

\section{UCDAI and clinical symptom scores}

There were no marked differences in UCDAI and clinical symptom (diarrhea, abdominal pain and mucous bloody stool) scores before treatment between both series, but UCDAI and clinical symptom scores decreased significantly postdrug exposure, and the decrease was more severe in combination group $(p<0.05)$.

\section{DISCUSSION}

Ulcerative colitis (UC) has a long course of treatment and a high incidence of relapse. Delayed treatment of UC may result in deterioration which may lead to intestinal perforation [9] and cancer [10], which endanger the lives of people. To date, the pathogenesis of $\mathrm{UC}$ is considered to be related to persistent intestinal infection, abnormal immune regulation, and intestinal mucosal barrier dysfunction. These factors highlight the clinical significance of the control of mucosal microflora for reduction of inflammatory reactions due to bacterial colonies [11].

Mesalazine, a 5-aminosalicylic acid preparation [12], is frequently used for UC treatment. It inhibits the secretion of colonic mucosa, decreases the secretion of prostaglandins and leukotrienes, inhibits the formation of OFRs, and suppresses the release of inflammatory mediators. It exerts a favorable anti-inflammatory effect, with few side effects, and it alleviates clinical symptoms such as abdominal pain, diarrhea, and pus and bloody stool, thereby restoring the colonic mucosa of patients $[13,14]$. Bifid Triple Viable Capsule (BTVC) is a mixture of three kinds of probiotics, namely, Bifidobacterium, Enterococcus and Lactobacillus acidophilus. It can cross the gastric acid barrier and reach the intestinal tract directly, thereby supplementing normal beneficial bacteria groups in the intestinal tract. This contributes to improving the intestinal flora environment, maintaining the intestinal microecological balance, and reducing intestinal endotoxin production $[15,16]$.

The results obtained in this study revealed no notable difference in levels of oxidative stress and inflammatory reaction factors between the two groups before treatment, but these indexes were markedly reduced in the combination group post-treatment, relative to control.

Table 3: Comparison of mucosal barrier indices between both groups

\begin{tabular}{lcccc}
\hline Variable & Control group (n=11) & Combination group $(\mathbf{n = 8 9})$ & $\boldsymbol{t}$ & $\boldsymbol{P}$-value \\
\hline D-lactic acid (mmol/L) & & & & \\
Before & $5.34 \pm 0.62$ & $5.41 \pm 0.59$ & 0.3693 & 0.713 \\
After & $4.68 \pm 0.41$ & $4.11 \pm 0.38$ & 4.6545 & $<0.001$ \\
DAO (U/L) & & & & \\
Before & $7.73 \pm 0.97$ & $7.68 \pm 0.91$ & 0.1707 & 0.865 \\
After & $6.54 \pm 0.87$ & $5.41 \pm 0.59$ & 5.6629 & $<0.001$ \\
ET (EU/mL) & & & & \\
Before & $0.24 \pm 0.09$ & $0.25 \pm 0.07$ & 0.4328 & 0.666 \\
After & $0.19 \pm 0.03$ & $0.14 \pm 0.02$ & 7.3665 & $<0.001$ \\
\hline
\end{tabular}

Table 4: Clinical symptom scores in the two groups

\begin{tabular}{lcccc}
\hline Variable & Control $(\mathbf{n = 1 1 )}$ & Combination $(\mathbf{n = 8 9})$ & $\boldsymbol{t}$ & $\boldsymbol{P}$-value \\
\hline UCDAl & & & & \\
Before & $7.62 \pm 1.02$ & $7.57 \pm 0.94$ & 0.1679 & 0.870 \\
After & $5.03 \pm 0.59$ & $3.68 \pm 0.43$ & 9.4087 & $<0.001$ \\
Diarrhea & & & \\
Before & $2.71 \pm 0.61$ & $2.66 \pm 0.63$ & 0.2491 & 0.804 \\
After & $1.66 \pm 0.34$ & $1.03 \pm 0.27$ & 7.0919 & $<0.001$ \\
Abdominal pain & $1.88 \pm 0.38$ & $1.84 \pm 0.41$ & 0.3075 & 0.759 \\
Before & $1.20 \pm 0.26$ & $0.88 \pm 0.11$ & 7.5124 & $<0.001$ \\
After & & & & \\
Mucous bloody stool & $4.42 \pm 0.68$ & $4.40 \pm 0.55$ & 0.1108 & 0.912 \\
Before & $2.55 \pm 0.58$ & $1.91 \pm 0.63$ & 3.2036 & 0.002 \\
After & & & & \\
\hline
\end{tabular}


The level of the inflammatory factor hs-CRP increases in different degrees in both active and remission stages of UC. Moreover, TNF- $\alpha$ is a critical pro-inflammatory cytokine which induces inflammatory reaction in the intestinal mucosa, leading to different degrees of injury in the intestinal mucosa. This underlies its important role in the pathogenesis of UC. In addition, IL-6 is a pro-inflammatory factor which induces pathological damage in colon mucosa and leads to inflammation of intestinal mucosa.

It has been reported that IL-10 is an inflammatory cytokine which activates monocytes and granulocytes to produce GM-CSF and G-CSF, and protects macrophages and neutrophils from chemotaxis [17]. In this study, after treatments, serum levels of hs-CRP, TNF- $\alpha$ and IL- 6 were decreased significantly, while serum IL-10 showed a significant increase.

Lipid peroxidation is key causative factor for UC. An increase in OFR content causes an inflammatory reaction. In patients with $U C$, the MDA contents are increased, while the activity of the antioxidant enzyme SOD decreases, and the OFR scavenging is limited. These changes further aggravate the deterioration of the patient's condition.

The results of this study show that the combination group displayed lower MDA content and higher SOD activity than the control group, implying that the combination therapy mitigated oxidative stress in patients. During UC treatment, the use of combination of Bifid Triple Viable Capsules and mesalazine further enhanced the OFR scavenging capacity of the patients, reduced MDA concentration, increased SOD activity, and decreased levels of inflammatory factors. Moreover, the combination treatment inhibited intestinal inflammatory reactions, abated intestinal mucosal cell injury, improved intestinal function and enhanced clinical efficacy [18]. In this study, UCDAI and clinical symptom scores were decreased significantly after treatment, and the decreases were statistically greater in combination drug-treated patients than in controls, suggesting that combination of mesalazine and Bifid Triple Viable Capsules resulted in a promising performance in the treatment of UC.

Impairment of mucosal barrier of the intestine is a major presentation in UC. Therefore, the repair of this impairment in patients is the crux of UC treatment. Studies have shown that LA is a reliable index of intestinal permeability, while $\mathrm{DAO}$ is an effective index of intestinal mucosal structural integrity, and ET is a valid indicator of intestinal function damage [19]. The present study revealed lower amounts of D-LA, DAO and ET in UC patients given combination treatment than in controls, suggesting that combination of BTVCs and mesalazine effectively regulated intestinal flora balance, reduced inflammatory factor infiltration and intestinal mucosal injury, and repaired intestinal mucosal barrier function.

\section{CONCLUSION}

The combined use of mesalazine and BTVCs significantly enhances treatment efficacy by mitigating inflammatory injury and oxidative stress reaction, as well as improving the intestinal micro-ecological environment of UC patients.

\section{DECLARATIONS}

\section{Conflict of Interest}

No conflict of interest associated with this work.

\section{Contribution of Authors}

We declare that this work was done by the authors named in this article and all liabilities pertaining to claims relating to the content of this article will be borne by the authors. Lina Wei and Hua Xu conceived and designed the study, drafted the manuscript, collected, analyzed and interpreted the experimental data. Lina Wei revised the manuscript for important intellectual content. Both authors read and approved the final manuscript.

\section{Open Access}

This is an Open Access article that uses a funding model which does not charge readers or their institutions for access and distributed under the terms of the Creative Commons Attribution License (http://creativecommons.org/licenses/by/ 4.0) and the Budapest Open Access Initiative (http://www.budapestopenaccessinitiative.org/rea d), which permit unrestricted use, distribution, and reproduction in any medium, provided the original work is properly credited.

\section{REFERENCES}

1. Ordás I, Eckmann L, Talamini M, Baumgart DC, Sandborn WJ. Ulcerative colitis. Lancet 2012; 380: 1606-1619.

2. Wędrychowicz A, Zając A, Tomasik P. Advances in nutritional therapy in inflammatory bowel diseases: Review. World J Gastroenterol 2016; 22: 1045-1066.

Trop J Pharm Res, October 2021; 20(10): 2217 
3. Neurath MF, Leppkes M. Resolution of ulcerative colitis. Semin Immunopathol 2019; 41: 747-756.

4. Lazar V, Ditu LM, Pircalabioru GG, Gheorghe I, Curutiu C, Holban AM, Picu A, Petcu L, Chifiriuc MC. Aspects of Gut Microbiota and Immune System Interactions in Infectious Diseases, Immunopathology, and Cancer. Front Immunol 2018; 9: 1830.

5. Gao J, Xu K, Liu H, Liu G, Bai M, Peng C, Li T, Yin Y. Impact of the Gut Microbiota on Intestinal Immunity Mediated by Tryptophan Metabolism. Front Cell Infect Microbiol 2018; 8: 13.

6. Datta $P$, Rewers-Felkins $K$, Kallem RR, Baker T, Hale TW. Determination of Mesalamine levels in Human Milk as a Function of Dose. Breastfeed Med 2019; 14: 98101.

7. Goldsmith JR, Waljee AK, George T, Brown A, Choe HM, Noureldin M, Bernstein SJ, Higgins PDR. 5-ASA to sulfasalazine drug switch program in patients with ulcerative colitis. Am J Manag Care 2018; 24: SP303SP308.

8. World Medical Association. World Medical Association Declaration of Helsinki: ethical principles for medical research involving human subjects. JAMA. 2013; 310: 2191-4.

9. DiCaprio D, Lee-Kong S, Stoffels G, Shen B, Al-Mazrou $A$, Kiran RP, Korelitz B, Swaminath A. Management of iatrogenic perforation during colonoscopy in ulcerative colitis patients: a survey of gastroenterologists and colorectal surgeons. Int J Colorectal Dis 2018; 33: 16071616.

10. Yao $D$, Dong $M$, Dai $C$, Wu S. Inflammation and Inflammatory Cytokine Contribute to the Initiation and Development of Ulcerative Colitis and Its Associated Cancer. Inflamm Bowel Dis 2019; 25: 1595-1602.

11. Shen ZH, Zhu CX, Quan YS, Yang ZY, Wu S, Luo WW, Tan B, Wang $X Y$. Relationship between intestinal microbiota and ulcerative colitis: Mechanisms and clinical application of probiotics and fecal microbiota transplantation. World J Gastroenterol 2018; 24: 5-14.
12. Kato S, Ishibashi A, Kani K, Yakabi K. Optimized Management of Ulcerative Proctitis: When and How to Use Mesalazine Suppository. Digestion 2018; 97: 59-63.

13. Sehgal $P$, Colombel JF, Aboubakr A, Narula $N$. Systematic review: safety of mesalazine in ulcerative colitis. Aliment Pharmacol Ther 2018; 47: 1597-1609.

14. Martinez CAR, Campos FG, Kanno DT, Meneses EC, Matijascic GM, Goto EFK, Pereira JA. Enemas with mesalazine increase the tissue contents of mucins in the colonic mucosa devoid of fecal stream. Acta Cir Bras 2019; 34: e201900406.

15. Gong J, Bai T, Zhang L, Qian W, Song J, Hou X. Inhibition effect of Bifidobacterium longum, Lactobacillus acidophilus, Streptococcus thermophilus and Enterococcus faecalis and their related products on human colonic smooth muscle in vitro. PLoS One 2017; 12: e0189257.

16. Chen MY, Qiu ZW, Tang HM, Zhuang KH, Cai QQ, Chen $X L, L i H B$. Efficacy and safety of bifid triple viable plus aminosalicylic acid for the treatment of ulcerative colitis: A systematic review and meta-analysis. Medicine (Baltimore) 2019; 98: e17955.

17. Gomes RG, Brito CAA, Martinelli VF, Santos RND, Gomes FODS, Peixoto CA, Crispim JO, Diniz GTN, Donadi EA, Lucena-Silva N. HLA-G is expressed in intestinal samples of ulcerative colitis and Crohn's disease patients and HLA-G5 expression is differentially correlated with TNF and IL-10 cytokine expression. Hum Immunol 2018; 79: 477-484.

18. Liu B, Piao X, Guo L, Liu S, Chai F, Gao L. Ursolic acid protects against ulcerative colitis via anti-inflammatory and antioxidant effects in mice. Mol Med Rep 2016; 13 : 4779-4785.

19. Yun HF, Liu R, Han D, Zhao X, Guo JW, Yan FJ, Zhang $C$, Sun HW, Liang GQ, Zhang GX. Pingkui Enema Alleviates TNBS-Induced Ulcerative Colitis by Regulation of Inflammatory Factors, Gut Bifidobacterium, and Intestinal Mucosal Barrier in Rats. Evid Based Complement Alternat Med 2020; 2020 : 3896948. 